\title{
Post-Exposure Prophylaxis and Methamphetamine Use among Young Sexual Minority Men: The P18 Cohort Study
}

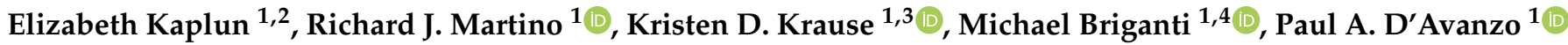 \\ and Perry N. Halkitis $1,2,3, *$
}

1 Center for Health, Identity, Behavior, and Prevention Studies, Rutgers School of Public Health, Newark, NJ 07102, USA; lizkaplun@gmail.com (E.K.); Richardjmartino@gmail.com (R.J.M.); kristen.krause@rutgers.edu (K.D.K.); mb1629@sph.rutgers.edu (M.B.); pad196@newark.rutgers.edu (P.A.D.)

2 Department of Epidemiology \& Biostatistics, Rutgers School of Public Health, Piscataway, NJ 08854, USA

3 Department of Urban-Global Public Health, Rutgers School of Public Health, Newark, NJ 07102, USA

4 Department of Health Behavior, Society, and Policy, Rutgers School of Public Health, Piscataway, NJ 08854, USA

* Correspondence: perry.halkitis@rutgers.edu

\section{check for}

Citation: Kaplun, E.; Martino, R.J.; Krause, K.D.; Briganti, M.; D'Avanzo, P.A.; Halkitis, P.N. Post-Exposure Prophylaxis and Methamphetamine Use among Young Sexual Minority Men: The P18 Cohort Study. Int. J. Environ. Res. Public Health 2022, 19, 712. https://doi.org/10.3390/ ijerph19020712

Academic Editor: Paul B.

Tchounwou

Received: 15 December 2021

Accepted: 5 January 2022

Published: 9 January 2022

Publisher's Note: MDPI stays neutral with regard to jurisdictional claims in published maps and institutional affiliations.

Copyright: (C) 2022 by the authors. Licensee MDPI, Basel, Switzerland. This article is an open access article distributed under the terms and conditions of the Creative Commons Attribution (CC BY) license (https:// creativecommons.org/licenses/by/ $4.0 /)$.

\begin{abstract}
Methamphetamine use is associated with increased risk of HIV infection among young sexual minority men (SMM). Post-exposure prophylaxis (PEP) is an effective strategy for individuals who are exposed to HIV, but there is limited research about PEP use among young SMM and its relationship with methamphetamine use. This study analyzes the association between ever PEP use and recent methamphetamine use among young SMM in New York City, using cross-sectional data from the P18 Cohort Study $(n=429)$. Multivariable logistic regression models were used to assess the association between methamphetamine use and ever PEP use. Compared with those who had not used methamphetamine in the last 6 months, young SMM who did use methamphetamine were significantly more likely to have ever used PEP (AOR = 6.07, 95\% CI: 2.10-16.86). Young SMM who had ever used PrEP had 16 times higher odds of ever using PEP (AOR = 16, 95\% CI: 7.41-35.95). Those who completed bachelor's degrees were $61 \%$ less likely to have ever used PEP (AOR $=0.39,95 \% \mathrm{CI}$ : 0.17-0.88). These data suggest that methamphetamine use could increase the risk of HIV infection, highlighting the critical need to target interventions for young SMM who use methamphetamine and are more likely to engage in unprotected intercourse.
\end{abstract}

Keywords: substance use; sexual health; sexual minority; gender minority; racial and ethnic minority; syndemic theory

\section{Introduction}

Rates of HIV infection have declined significantly over the last decade, but disparities persist among vulnerable populations. The Center for Disease Control and Prevention (CDC) reports that approximately 36,400 people were newly infected with HIV in the United States in 2018 [1]. Of these new diagnoses, $69 \%$ were men who have sex with men (MSM) or sexual minority men (SMM), with young SMM aged $13-24$ compromising $80 \%$ of new diagnoses in their age group, and 25\% of all SMM [2]. Additionally, racial and ethnic minority men are disproportionately affected by HIV.

Among young SMM diagnosed with HIV in 2018 (ages 13-24), 47\% were non-Hispanic (NH) Black, 31\% were Hispanic/Latino, and 15\% were NH White [2].

Although there has been much progress since the height of the AIDS epidemic in the mid-1980s, progress has stalled in recent years, with nearly constant annual incidence rates since 2014 [1]. In the last few decades, HIV has become a chronic condition that is highly manageable through antiretroviral therapy [3], and in 2012, the U.S. Food and Drug Administration approved Truvada ${ }^{\circledR}$, the first pre-exposure prophylaxis (PrEP) medication for people who are HIV negative but are at high risk of contracting HIV [4]. This was a 
monumental medical achievement for HIV prevention, and has proven to be highly effective when used appropriately [4]. Recent studies, however, suggest that while many have heard of PrEP, uptake among SMM has been low, only about 3.4\% ever using it, particularly among racial and ethnic minorities, who are at higher risk of HIV infection $[2,5,6]$.

On the other hand, post-exposure prophylaxis (PEP) is a drug treatment administered once daily for 28 days, meant to prevent HIV infection after exposure [7]. It is also highly effective in preventing HIV, but it is not as well-known as PrEP, and is generally even more underutilized. Current literature about PEP uptake is limited, but one study examining PEP use reported that $7.4 \%$ of participants in a New York City, NY (NYC) sample composed of young SMM, transgender women, and cisgender women of color, had ever used PEP, and only $13.2 \%$ were aware of PEP [8]. If taken for 28 days and initiated within 72 hours of exposure to HIV, PEP can reduce the odds of infection by $81 \%$ [9]. This underutilization of PEP can be partially explained by barriers such as lack of awareness, stigma, and access among high-risk populations [8].

In recent years, individuals who engage in sexualized drug use, or "chemsex," behaviors have emerged as a potentially vulnerable group at risk for HIV infection [10]. In particular, methamphetamine use has been associated with high-risk sexual behaviors and HIV infection among SMM [11]. Due to these associations in emerging young SMM, it is crucial to understand current PEP uptake in this population to better implement and cater education and outreach for HIV prevention for high-risk populations.

Methamphetamine is a strong and addictive central nervous system stimulant that can elevate one's energy, alertness, concentration, and mood; however, it is also associated with depression, anxiety, psychosis, violent behavior, and overall poor health outcomes [12]. It is also referred to as crystal meth, chalk, ice, and can be orally administered, smoked, injected, snorted, and "booty bumped" (administered rectally) [12,13]. Methamphetamine use has been associated with significant public health problems in the U.S. for over 35 years, with little consensus over how large of an issue it really is. A literature review conducted by Gonzales et al. asserted that national-based surveys depict methamphetamine use as a minor concern, but do not capture the true extent of the issue, whereas data sources from law-enforcement groups, welfare agencies, and substance abuse treatment programs indicate a much more significant problem [11].

For sexual minority individuals, rates of methamphetamine use have decreased for those aged 18-25 from 2017 to $2018(-0.7 \%)$, but there has been a resurgence for those aged 26 and older $(+1.4 \%)$ [14]. These national statistics are important to consider, but they do not shed light on the differences among various regions in the U.S. For instance, a study conducted in NYC showed that while methamphetamine use declined significantly among SMM throughout the 2000s after public health initiatives aimed to reduce methamphetamine use in this subgroup [15], there has been a resurgence since 2011, particularly among SMM communities of color [16]. Another study examined the patterns of methamphetamine use among young MSM in Chicago, Illinois, and found that $13 \%$ of participants $(n=310)$ aged 16-24 reported methamphetamine use [17].

These current trends of methamphetamine use are concerning for young SMM emerging into adulthood, who may struggle with psychological distress, self-esteem, and perceived stigma associated with their sexual identity. Research suggests a link between these issues and young SMM partnering with older men in adult social settings, where substance use and casual sexual encounters are more common [17]. Unprotected sex and sexual risk-taking behaviors increase the risk of HIV transmission and infection among SMM. Methamphetamine use is strongly associated with sexual risk-taking as well, and research shows that this stimulant elevates levels of impulsivity, desires to engage in unprotected sex, and sex with more than one and/or anonymous partners [18,19].

Overall, the emerging literature about methamphetamine use in SMM populations and the high risk of HIV infection indicates a growing need for more research and the implementation of science to better focus primary and secondary prevention efforts. Although PrEP is a highly effective preventative measure young SMM can take to significantly lower 
their risk of HIV infection, uptake is reported to be quite low. In a NYC survey, 41\% of SMM had heard of PrEP, but only 3.4\% of the sample had ever taken it [5]; in general, PrEP uptake rates are lower among young SMM than older SMM [20]. Among SMM, researchers found that PrEP awareness was significantly lower for methamphetamine users when compared with those who do not use methamphetamines ( $89 \%$ and $95 \%$, respectively, $p=0.01)$, but there was no significant difference in actual PrEP use between the two groups [21].

The current literature about PEP and methamphetamine use in the young SMM population is extremely limited. One retrospective cohort study sought to evaluate any association between PEP prescriptions at a community health center in Boston, MA and methamphetamine use during the HIV exposure event and chronic methamphetamine use. This study found that frequent methamphetamine users came back for repeat PEP prescriptions more than non-methamphetamine users (AOR $=5.13,95 \% \mathrm{CI}: 2.82-9.34)$ but were significantly more likely to seroconvert during follow-up periods (AHR $=3.61)$. Additionally, methamphetamine use was associated with increased odds of unprotected anal intercourse (AOR $=2.12,95 \% \mathrm{CI}: 1.16-3.87)$ [22].

It has also been documented that healthcare providers may be hesitant to prescribe PEP to stimulant users, due to observations of poor compliance with the therapy among methamphetamine users, with significantly lower rates of PEP course completion when compared with non-substance users [23,24]. The public health implications of these medical decisions could be detrimental to young SMM who use methamphetamine, as they are more likely to engage in sexual risk-taking behavior and unprotected sex [25].

Due to the aforementioned gaps in the literature, this study seeks to understand individual level factors associated with young SMM who have ever used PEP. The aim of this study was to evaluate the association of ever PEP use and methamphetamine use (within the last 6 months) among young YSMM living in the New York City metropolitan area (NYC). It was hypothesized that there was a significant association between those who reported using methamphetamines in the past 6 months (at time of follow-up) and using PEP at least once ever.

\section{Materials and Methods}

\subsection{Study Design and Data Sources}

The data for this cross-sectional analysis were drawn from the baseline of the second wave of the P18 Cohort Study, a prospective cohort study that surveyed young sexual minority men (YSMM) in NYC to assess the syndemic of substance use, mental health burden and HIV. Study details were previously published [26] and are briefly summarized here. Briefly, recruitment for the study was conducted in two waves, Wave I (2009-2014) recruited a total of 600 young SSM between the ages of 18-19, and Wave II (2014-2019) included participants from the original cohort and new age-matched participants for a total of $n=665$ participants between the ages of 22 and 23. Participants were recruited through a number of active and passive methods, including in-person events (e.g., PRIDE festivals, community meetings), online methods (e.g., mobile applications and websites), and referrals from other participants. At each study visit (every six months), audio computer-assisted self-interviews (ACASI) were used for participants to provide details on sociodemographic factors, psychosocial factors, and drug use. Participants also completed a timeline follow back (TLFB), a calendar-based, semi-structured interview used to evaluate event-level behavioral data for the 30-day period before the assessment to assess sexual behavior [27]. Study participants were also tested for HIV serostatus with a rapid finger prick antibody test. Finally, participants were compensated at each study visit. 


\subsection{Study Participants}

Young sexual and gender minority young adults who were assigned male at birth ( $n=665)$ were recruited for the P18 Cohort Study. Participants were eligible for the study if their HIV status was negative or unknown at the time of screening, if they were age 22-23, and assigned male at birth. Participants also must have reported having sex with a man in the last 6 months at the time of screening.

\subsection{PrEP and PEP Use}

Ever PrEP use was determined through the question "have you ever taken PrEP?" Participants were provided with the definition of PrEP as "an HIV-negative person taking a daily pill to prevent HIV" when answering this question. Ever PEP use was determined through the question "have you ever taken PEP?"

\subsection{Methamphetamine Use}

To determine ever methamphetamine use in the last 6 months, all forms of use were categorized into one variable. The questions that were combined were: "In the past 6 months, have you injected crystal, ice or tina (methamphetamine)?"; "In the past 6 months, have you smoked/snorted crystal (meth)?"; "In the past 6 months, have you booty bumped crystal (meth)?" These questions were used to create a binary variable if a participant had used methamphetamines through any route of administration in the last 6 months (at time of follow-up).

\subsection{Unprotected Sexual Behavior}

Participants were asked about various unprotected sexual behavior, capturing the number of given and/or received unprotected sexual acts the participants performed in the last month using the TLFB. These behaviors were: total unprotected oral acts given (past month), total unprotected oral acts received (past month), total unprotected anal acts given (past month), total unprotected anal acts receptive (past month), total unprotected mutual masturbation acts (past month), total unprotected rimming acts given (past month), total unprotected rimming acts received (past month), and total unique partners (past month).

\subsection{Sociodemographic Characteristics}

All sociodemographic data was self-reported by the participant. There were six racial/ethnic groups in this study consisting of Hispanic/Latino, Non-Hispanic (NH) Black, NH Asian, NH Mixed, NH Native American/Other, and NH White. Nation of birth was dichotomized into US-born and foreign-born. Sexual identity was determined by using the Kinsey scale by asking participants "Do you identify as homosexual?" with responses ranging from identifying as not exclusively homosexual (0) to exclusively homosexual (6) [28]. These responses were dichotomized for this study as exclusively homosexual versus not exclusively homosexual. Total income was self-reported by participants with a 12-category response set, but was then recoded into "Low" (<\$5000), "Middle" (\$5000-19,999), and "Upper" ( $\geq \$ 20,000)$ income categories, to match a previously published study using the same dataset [29]. Education status was categorized into four groups of educational attainment: high school completion or lower, associate degree, bachelor's degree, and graduate degree. Finally, participants were asked about their HIV status, and their responses were confirmed through rapid antibody testing by finger prick. Participants from Wave I who tested positive for HIV had previously received viral load testing, as well.

\subsection{Analytic Plan}

The software R and RStudio (version 3.6.1) were used for three levels of data analysis. First, descriptive statistics were generated for all measures. Second, chi-squared bivariate analyses were performed on the frequency of sociodemographic factors, PrEP use, methamphetamine use in the last 6 months by PEP use, and risky sexual behaviors. Statistical significance was set at two-tailed $\alpha<0.05$. For some analyses, Fisher's Test was 
used in place of chi-squared testing, due to expected cell sizes under 5. Measures that were statistically significant in the bivariate tests were included in the full multivariable logistic regression model. Third, the association between using methamphetamine in the past 6 months as the main exposure variable and ever using PEP as the main outcome variable was determined through univariate logistic regression modeling in the main unadjusted model; univariate logistic regression models for demographic and behavior measures were also used to assess association with PEP use individually. Finally, an adjusted multivariate logistic regression model was created for ever use of PEP (reference $=$ no) as the dependent variable and ever using methamphetamine in the past 6 months as the main independent variable. The final model controlled for race/ethnicity because of priori knowledge [1], as well as educational attainment and PrEP ever use because of statistical significance in the bivariate analyses.

\section{Results}

\subsection{Sample Characteristics}

Table 1 shows the characteristics of the baseline visit for all participants for the second wave of the P18 cohort, conducted at 42 months after the beginning of the first wave. The baseline sample was racially and ethnically diverse, with $31.88 \%$ of the participants identifying as Hispanic/Latino, 25.56\% identifying as non-Hispanic Black, 25.26\% identifying as non-Hispanic White, 7.37\% identifying as non-Hispanic Asian, 6.47\% identifying as non-Hispanic multiracial, and 3.46\% identifying as non-Hispanic Native American or "other". Most participants were born in the United States (84.21\%), and identified their sexual orientation as exclusively homosexual (82.26\%). For annual personal income, 32.93\% of participants reported a low annual income, $30.08 \%$ reported a middle annual income, and $32.18 \%$ reported an upper annual income. Only $33.4 \%$ of participants were currently enrolled in school at the time of the visit, and $47.8 \%$ reported having a high school diploma or less. Participants were mostly HIV negative (95.59\%). Only 6.47\% of participants reported using methamphetamine in the last 6 months; $8.87 \%$ reported using PrEP ever; and $8.57 \%$ reported using PEP ever.

Table 1. Characteristics of P18 Cohort Study participants from baseline Wave II sample $(n=665)$.

\begin{tabular}{|c|c|c|}
\hline Variable & $n$ & $\%$ \\
\hline Race/Ethnicity & 665 & 100 \\
\hline Hispanic/Latino & 212 & $31.88 \%$ \\
\hline Black Non-Hispanic & 170 & $25.56 \%$ \\
\hline Asian Non-Hispanic & 49 & $7.37 \%$ \\
\hline Mixed Non-Hispanic & 43 & $6.47 \%$ \\
\hline Native American/Other Non-Hispanic & 23 & $3.46 \%$ \\
\hline White Non-Hispanic & 168 & $25.26 \%$ \\
\hline Born in the U.S. & - & - \\
\hline No & 104 & $15.64 \%$ \\
\hline Yes & 560 & $84.21 \%$ \\
\hline Missing & 1 & $0.15 \%$ \\
\hline Sexual orientation & - & - \\
\hline Identifies as "not exclusively homosexual" & 118 & $17.74 \%$ \\
\hline Identifies as "exclusively homosexual" & 547 & $82.26 \%$ \\
\hline Annual personal income & - & - \\
\hline Lower $(<5 \mathrm{k})$ & 219 & $32.93 \%$ \\
\hline Middle (5 k-19,999) & 200 & $30.08 \%$ \\
\hline Upper $(>20 \mathrm{~K})$ & 214 & $32.18 \%$ \\
\hline Missing & 32 & $4.81 \%$ \\
\hline Current school enrollment & - & - \\
\hline No & 443 & $66.62 \%$ \\
\hline Yes & 222 & $33.38 \%$ \\
\hline
\end{tabular}


Table 1. Cont.

\begin{tabular}{ccc}
\hline Variable & $\boldsymbol{n}$ & $\boldsymbol{\%}$ \\
\hline Highest level of education completed & - & - \\
High school diploma or less & 318 & $47.82 \%$ \\
Associate degree & 78 & $11.73 \%$ \\
Bachelor's degree & 260 & $39.10 \%$ \\
Graduate degree & 8 & $1.20 \%$ \\
Missing & 1 & $0.15 \%$ \\
HIV Status & - & - \\
HIV- & 629 & $94.59 \%$ \\
HIV+ & 30 & $4.51 \%$ \\
Test not given & 6 & $0.90 \%$ \\
Nothamphetamine Use (In the past 6 months) & - & - \\
Nos & 617 & $92.78 \%$ \\
Missing & 43 & $6.47 \%$ \\
PrEP Use & 5 & $0.75 \%$ \\
No & - & - \\
Yes & 459 & $69.02 \%$ \\
Missing & 59 & $8.87 \%$ \\
PEP Use & 147 & $22.11 \%$ \\
No & - & - \\
Yes & 439 & $66.02 \%$ \\
Missing & 57 & $8.57 \%$ \\
\hline
\end{tabular}

\subsection{Bivariate Analysis}

The bivariate relationships between our variables are reported in Table 2, showing frequencies and percentages of PEP status by individual characteristics. Of 665 participants in the Wave II baseline group, there were 236 participants that were removed from the analytic sample because of missing values, most from the PEP and PrEP questions. Our final sample included 429 participants who reported whether they had ever taken PEP. There were no statistically significant differences in PEP use based on race/ethnicity, nation of birth, exclusive homosexual identity, income, school enrollment, or HIV status. There were differences in PEP use by education level, with those who completed a bachelor's degree having significantly lower frequencies of ever having taken PEP $(p=0.03)$. Ever having taken PrEP was significantly associated with ever having taken PEP $(p<0.0001)$. Finally, reporting having taken any form of methamphetamine in the last 6 months (at time of follow-up) was significantly associated with ever having taken PEP $(p=0.002)$.

Table 2. PEP ever use by participant characteristics, PrEP ever use, and recent methamphetamine use $(n=429)^{\mathrm{a}}$.

\begin{tabular}{|c|c|c|c|c|c|}
\hline & \multicolumn{4}{|c|}{ PEP Ever Use } & \multirow[b]{3}{*}{$p$-Value $(\alpha=0.05)^{b}$} \\
\hline & \multicolumn{2}{|c|}{ No $(n=378)$} & \multicolumn{2}{|c|}{ Yes $(n=51)$} & \\
\hline & $n$ & $\%$ & $n$ & $\%$ & \\
\hline Race/Ethnicity & & & & & $0.13^{\mathrm{b}}$ \\
\hline Hispanic/Latino & 125 & $90.58 \%$ & 13 & $9.42 \%$ & \\
\hline Black NH & 76 & $80.85 \%$ & 18 & $19.15 \%$ & \\
\hline Asian NH & 21 & $80.77 \%$ & 5 & $19.23 \%$ & \\
\hline Mixed NH & 24 & $88.89 \%$ & 3 & $11.11 \%$ & \\
\hline Native American/Other NH & 12 & $92.31 \%$ & 1 & $7.69 \%$ & \\
\hline White NH & 120 & $91.60 \%$ & 11 & $8.40 \%$ & \\
\hline Born in the U.S. & & & & & 0.54 \\
\hline No & 53 & $91.38 \%$ & 5 & $8.62 \%$ & \\
\hline Yes & 325 & $87.60 \%$ & 46 & $12.40 \%$ & \\
\hline
\end{tabular}


Table 2. Cont.

\begin{tabular}{|c|c|c|c|c|c|}
\hline & \multicolumn{4}{|c|}{ PEP Ever Use } & \multirow[b]{3}{*}{$p$-Value $(\alpha=0.05)^{\mathrm{b}}$} \\
\hline & \multicolumn{2}{|c|}{ No $(n=378)$} & \multicolumn{2}{|c|}{ Yes $(n=51)$} & \\
\hline & $n$ & $\%$ & $n$ & $\%$ & \\
\hline Sexual orientation & & & & & 0.83 \\
\hline Identifies as "not exclusively homosexual" & 51 & $86.44 \%$ & 8 & $13.56 \%$ & \\
\hline Identifies as "exclusively homosexual" & 327 & $88.38 \%$ & 43 & $11.62 \%$ & \\
\hline Annual Personal Income & & & & & 0.18 \\
\hline Lower $(<5 \mathrm{k})$ & 130 & $92.20 \%$ & 11 & $7.80 \%$ & \\
\hline Middle (5 k-19,999) & 105 & $85.37 \%$ & 18 & $14.63 \%$ & \\
\hline Upper $(>20 \mathrm{~K})$ & 143 & $86.67 \%$ & 22 & $13.33 \%$ & \\
\hline Current school enrollment & & & & & 0.36 \\
\hline No & 251 & $89.32 \%$ & 30 & $10.68 \%$ & \\
\hline Yes & 127 & $85.81 \%$ & 21 & $14.19 \%$ & \\
\hline Highest level of education completed & & & & & $0.027^{b}$ \\
\hline High school diploma or less & 162 & $85.26 \%$ & 28 & $14.74 \%$ & \\
\hline Associate degree & 38 & $84.44 \%$ & 7 & $15.56 \%$ & \\
\hline Bachelor's degree & 174 & $92.55 \%$ & 14 & $7.45 \%$ & \\
\hline Graduate degree & 4 & $66.67 \%$ & 2 & $33.33 \%$ & \\
\hline HIV Status & & & & & $0.40^{\mathrm{b}}$ \\
\hline HIV- & 375 & $88.24 \%$ & 50 & $11.76 \%$ & \\
\hline HIV+ & 3 & $75.00 \%$ & 1 & $25.00 \%$ & \\
\hline PrEP Ever Use & & & & & $<0.0001$ \\
\hline No & 352 & $92.63 \%$ & 28 & $7.37 \%$ & \\
\hline Yes & 26 & $53.06 \%$ & 23 & $46.94 \%$ & \\
\hline Methamphetamine Use (In the past 6 months) & & & & & $0.0015^{b}$ \\
\hline No & 361 & $89.58 \%$ & 42 & $10.42 \%$ & \\
\hline Yes & 17 & $65.38 \%$ & 9 & $35.62 \%$ & \\
\hline
\end{tabular}

a There were 236 participants with missing values removed from the analytic sample. ${ }^{b}$ Fisher's exact test was used for analysis instead of $\chi^{2}$.

T-tests were run for high-risk sexual behaviors and PEP ever use, but there were no significant associations present, and therefore they were not included in the multivariable analysis (Appendix A).

\subsection{Multivariable Analysis}

Crude models examining ever PEP use by individual characteristics, ever PrEP use, and recent methamphetamine use were fit to determine which variables were significantly associated with the outcome variable. In the univariate analyses, only race/ethnicity, educational attainment, PrEP ever use, and methamphetamine use (in the last 6 months) were significant at $\alpha=0.05$, shown in Table 3 . These same measures, however, were included in the multivariate logistic regression model because of a priori knowledge or bivariate analysis significance (Table 2). 
Table 3. Binary logistic regression examining associations between ever PEP use and individual characteristics, PrEP use, and methamphetamine use.

\begin{tabular}{|c|c|c|c|c|}
\hline Variable & OR $(95 \% \mathrm{CI})$ & $p$-Value & AOR $(95 \% \mathrm{CI})$ & $p$-Value \\
\hline \multicolumn{5}{|l|}{ Race/Ethnicity } \\
\hline White (Reference) & 1 & & 1 & \\
\hline Hispanic/Latino & $1.13(0.49-2.68)$ & 0.77 & $1.01(0.38-2.69)$ & 0.99 \\
\hline Black NH & $2.58(1.17-5.93)$ & 0.02 & $2.47(0.94-6.74)$ & 0.07 \\
\hline Asian NH & $2.6(0.76-7.96)$ & 0.11 & $3.16(0.75-12.04)$ & 0.10 \\
\hline Mixed NH & $1.43(0.31-4.99)$ & 0.61 & $1.27(0.23-5.44)$ & 0.76 \\
\hline Native American/Other NH & $0.91(0.05-5.33)$ & 0.93 & $0.45(0.02-3.44)$ & 0.51 \\
\hline \multicolumn{5}{|l|}{ Highest level of education completed } \\
\hline High school diploma or less (Reference) & 1 & & 1 & \\
\hline Associate degree & $1.06(0.40-2.49)$ & 0.9 & $1.34(0.44-3.63)$ & 0.58 \\
\hline Bachelor's degree & $0.46(0.23-0.90)$ & 0.03 & $0.39(0.17-0.88)$ & 0.03 \\
\hline Graduate degree & $2.88(0.39-15.47)$ & 0.24 & $1.93(0.20-14.35)$ & 0.53 \\
\hline \multicolumn{5}{|l|}{ Methamphetamine Use } \\
\hline Yes & $4.54(1.83-10.62)$ & 0.0007 & $6.07(2.10-16.86)$ & 0.0006 \\
\hline \multicolumn{5}{|l|}{ PrEP Ever Use } \\
\hline Yes & $11.09(5.63-22.06)$ & $<0.0001$ & $16.0(7.41-35.95)$ & $<0.001$ \\
\hline
\end{tabular}

$\mathrm{CI}=$ Confidence Interval. $\mathrm{OR}=$ Odds Ratios. $\mathrm{AOR}=$ Adjusted Odds Ratios.

While not significant in the bivariate model, race was a significant factor in the crude regression model. Specifically, non-Hispanic Black identity was significantly associated with greater odds of ever using PEP (OR $=2.58,95 \% \mathrm{CI}$ : 1.17-5.93); no race or ethnicity, however, had significant associations to PEP use in the adjusted model. Those who had ever used PrEP were 16 times more likely to have ever used PEP (95\% CI: 7.41-35.95, $p<0.0001$ ). Additionally, participants who reported using methamphetamines in the last 6 months (at time of follow-up) had 6.07 times the odds of ever using PEP (95\% CI: 2.10-16.86, $p=0.0006)$. Conversely, those who completed bachelor's degrees were $61 \%$ less likely to have ever used PEP than those with just a high school education $(\mathrm{AOR}=0.39,95 \% \mathrm{CI}$ : $0.17-0.88)$.

\section{Discussion}

\subsection{Methamphetamine Use}

This study suggests that recent methamphetamine use is significantly associated with ever using PEP among young SMM in the NYC area, when compared with those who had not recently used methamphetamines. As one of the first studies to assess this association, these findings shed light on young methamphetamine users that are more likely to use a proven HIV prevention method. Stimulant use has been associated with unprotected intercourse and intercourse with HIV positive individuals, particularly among young SMM across many studies $[17,18,25]$, which makes them a vulnerable population in need of resources such as PEP to prevent seroconversion. This study's findings contribute to this research, suggesting that methamphetamine use is an integral part of the interaction between high-risk sexual behavior and exposure to HIV among young SMM, as suggested by the literature. These findings, however, positively suggest that PEP is being utilized in the methamphetamine-using community to mitigate the risk of contracting HIV.

\subsection{PrEP Use}

The findings also revealed that participants who self-reported ever using PrEP had 16 times greater odds for ever using PEP $(p<0.001)$. This finding suggests that participants who have used PrEP have knowledge of and access to PEP, which is a positive outcome from outreach and prevention efforts dedicated to young SMM. However, more research needs to be done to understand if either form of biomedical HIV protection is predictive of using the other. 


\subsection{Education}

Another interesting finding from this study shows that participants who reported having a bachelor's degree as their highest level of educational attainment reported significantly less ever use of PEP when compared with participants with a high school diploma or less as their highest level of educational attainment (Table 3). There is currently no evidence that educational attainment is associated with PEP uptake, and it is unclear whether this relationship is because those with bachelor's degrees have less awareness around PEP use or because they are more likely to practice safer sex, and therefore do not seek PEP treatment. More research should be conducted to further understand the individual level characteristics regarding educational attainment as a predictor for PEP use.

\subsection{Race and Ethnicity}

While non-Hispanic Black participants were the only racial/ethnic category more likely to use PEP in a crude logistic regression model, this relationship was not significant after adjusting for methamphetamine use, educational attainment, and PrEP use. Black SMM, however, are disproportionately affected by HIV, and methamphetamine use is a risk factor of interest in understanding sexual risk-taking behavior, although the relationship remains unclear in black populations [30,31]. Jerome and Halkitis (2009) noted that there are strong associations between stigmatization, stress, and substance use within the black SMM community, which can stem from perceived pressure from family members or key figures to present as cisgender and/or heterosexual from a young age [31]. Later on in life, this stigmatization can lead to recovering a sense of belonging and family in the SMM drug-using community [31]. While the association between non-Hispanic Black participants and PEP use in this study does not maintain significance after adjusting for confounders, it is still very important to note that there is a strong initial association, because of a priori literature confirming a strong association to methamphetamine use and HIV seroconversion in the Black SMM community. There is currently no prior literature examining the relationship between race/ethnicity and PEP use, and more research should be conducted to evaluate the disparities in HIV prevention treatments among racial and ethnic groups.

\subsection{Strengths and Limitations}

This study revealed emerging associations that are consistent with the current literature about methamphetamine use and HIV prophylaxis use among young SMM. Additionally, this study sheds light on current trends in a specific sample in NYC which can, in turn, provide direction for public health campaigns and HIV prevention efforts. This study also had several limitations. First, the data was self-reported and did not use biomarker testing to measure recent methamphetamine use, which could allow for recall bias in the participants responses. Although the P18 Cohort Study used longitudinal collection that evaluated responses every 6 months, this analysis was cross-sectional in nature, using only one set of data collected at the start of Wave II, meaning that we cannot determine causality. The dataset was also rather small; 236 respondents with missing data were removed from the data for a total sample size of $n=429$. Additionally, because participants were comprised of a very limited group of people-those who were aged 22-23 at the time-the significance of factors such as income and educational attainment were limited. As the sample was so young, many of them did not have a substantial yearly income to report, which makes it difficult to examine the role of socio-economic status. Although the scope of the study sought to understand emerging patterns in methamphetamine and PEP use among young SMM as they enter adulthood, future research should recruit a wider range of participants with regard to age. Finally, because of the limited geographic region and age range, our results may not be generalizable across other areas in the U.S. 


\subsection{Public Health Implications}

The strong association between people who have taken PrEP and have ever taken PEP could be due to the fact that there is more knowledge and access for those who are already familiar with certain prophylaxis medications. If an at-risk person is seeing a primary care provider consistently to ensure they are taking the necessary precautions with PrEP, they could be more likely to also try PEP if they are ever in an emergency situation. On the other hand, the association between methamphetamine and PEP use could mean that participants who use methamphetamine are more likely to engage in unprotected sex or not use PrEP. This information can be used to increase public health campaigns targeting young SMM who use methamphetamine to increase primary and secondary HIV prevention efforts, specifically educating and providing community outreach about using biomedical prophylaxis therapies, while also encouraging safer sex.

Recent interventions to reduce HIV transmission among methamphetamine-using SMM have focused on understanding psychosocial factors and social structures through cognitive behavioral therapy [32]. Further research is necessary to understand the underpinnings and motivations behind PEP uptake and the demographic and behavioral patterns associated with methamphetamine use-particularly in at-risk groups such as young SMM in NYC-in order to develop effective interventions.

\section{Conclusions}

The purpose of this study was to evaluate and understand what demographic and behavioral factors are associated with ever use of PEP among a young population of SMM, and particularly recent methamphetamine use in any form. Our findings suggest that methamphetamine use was a significant predictor for ever using PEP in an emerging population of young SSM at risk for HIV infection. This study highlights the critical need for increasing public health interventions among vulnerable and high-risk populations in the NYC area.

Author Contributions: Conceptualization, E.K. and R.J.M.; methodology, E.K. and R.J.M.; software, E.K.; validation, E.K., R.J.M., P.A.D., and P.N.H.; formal analysis, E.K..; investigation, R.J.M., K.D.K., and P.A.D.; resources, P.N.H.; data curation, R.J.M.; writing-original draft preparation, E.K., R.J.M., K.D.K., and M.B.; writing-review and editing, P.A.D. and P.N.H.; visualization, E.K. and R.J.M.; supervision, K.D.K. and P.N.H.; project administration, P.A.D. and P.N.H.; funding acquisition, P.N.H. All authors have read and agreed to the published version of the manuscript.

Funding: Research reported in this publication was supported by the National Institute on Drug Abuse of the National Institutes of Health under Award Numbers 1R01DA025537 and 2R01DA025537. The content is solely the responsibility of the authors and does not necessarily represent the official views of the National Institutes of Health.

Institutional Review Board Statement: The study was conducted according to the guidelines of the Declaration of Helsinki, and approved by the Institutional Review Board of New York University (protocol \#10-6802, initially approved 10/20/2014).

Informed Consent Statement: Informed consent was obtained from all subjects involved in the study.

Data Availability Statement: To protect the confidentiality of P18 cohort participants, we do not make data freely available. Data are housed at the Rutgers School of Public Health's Center for Health, Identity, Behavior and Prevention Studies (CHIBPS), where the P18 cohort PI (P.H.) is housed.

Acknowledgments: The authors would like to thank the participants of this study as well as the staff and student interns at the Center for Health, Identity, Behavior, and Prevention Studies (CHIBPS) at the Rutgers School of Public Health.

Conflicts of Interest: The authors declare no conflict of interest. 


\section{Appendix A}

Table A1. Unprotected Sexual Behavior by PEP Use.

\begin{tabular}{|c|c|c|c|c|c|}
\hline & \multicolumn{2}{|c|}{ PEP Use Ever-No } & \multicolumn{2}{|c|}{ PEP Use Ever-Yes } & \multirow[b]{2}{*}{$p$-Value } \\
\hline & Mean & Standard Deviation & Mean & Standard Deviation & \\
\hline Total unprotected oral given & 3.43 & 4.39 & 4.14 & 4.45 & 0.26 \\
\hline Total unprotected oral received & 3.51 & 4.27 & 4.35 & 4.28 & 0.16 \\
\hline Total unprotected anal insertive & 0.87 & 2.65 & 1.37 & 2.75 & 0.20 \\
\hline Total unprotected anal receptive & 1.05 & 2.49 & 1.23 & 2.54 & 0.62 \\
\hline Total unprotected vaginal insertive & 0.01 & 0.16 & 0.00 & 0.00 & 0.13 \\
\hline Total unprotected mutual masturbation & 2.31 & 3.31 & 3.46 & 5.82 & 0.15 \\
\hline Total unprotected rimming received & 1.27 & 2.74 & 1.56 & 3.12 & 0.50 \\
\hline Total unprotected rimming given & 1.06 & 2.72 & 1.40 & 3.05 & 0.42 \\
\hline Total unprotected fingering received & 1.07 & 2.66 & 0.91 & 2.08 & 0.60 \\
\hline Total unprotected fingering given & 0.79 & 2.22 & 1.44 & 2.84 & 0.10 \\
\hline Unprotected fisting received & 0.01 & 0.15 & 0.02 & 0.13 & 0.66 \\
\hline Unprotected fisting insertive & 0.01 & 0.18 & 0.05 & 0.29 & 0.33 \\
\hline Total Unique partners & 2.48 & 2.76 & 3.33 & 3.67 & 0.09 \\
\hline
\end{tabular}

\section{References}

1. CDC. Estimated HIV Incidence and Prevalence in the United States, 2014-2018; Centers for Disease Control and Prevention: Atlanta, GA, USA, 2020.

2. CDC. Diagnoses of HIV Infection in the United States and Dependent Areas, 2018; Centers for Disease Control and Prevention: Atlanta, GA, USA, 2020.

3. Hong, F.F.; Mellors, J.W. Impact of Antiretroviral Therapy on HIV-1 Persistence: The Case for Early Initiation. AIDS Rev. 2015, 17, 71-82. [PubMed]

4. CDC. CDC Statement on FDA Approval of Drug for HIV Prevention. Available online: https://www.cdc.gov/nchhstp/ newsroom/2012/fda-approvesdrugstatement.html (accessed on 26 March 2021).

5. Marks, S.J.; Merchant, R.C.; Clark, M.A.; Liu, T.; Rosenberger, J.G.; Bauermeister, J.; Mayer, K.H. Potential Healthcare Insurance and Provider Barriers to Pre-Exposure Prophylaxis Utilization Among Young Men Who Have Sex with Men. AIDS Patient Care STDs 2017, 31, 470-478. [CrossRef] [PubMed]

6. $\quad$ Pérez-Figueroa, R.E.; Kapadia, F.; Barton, S.C.; Eddy, J.A.; Halkitis, P.N. Acceptability of PrEP Uptake Among Racially/Ethnically Diverse Young Men Who Have Sex With Men: The P18 Study. AIDS Educ. Prev. 2015, 27, 112-125. [CrossRef] [PubMed]

7. Smith, D.K.; Grohskopf, L.A.; Black, R.J.; Auerbach, J.D.; Veronese, F.; Struble, K.A.; Cheever, L.; Johnson, M.; Paxton, L.A.; Onorato, I.M.; et al. Antiretroviral postexposure prophylaxis after sexual, injection-drug use, or other nonoccupational exposure to HIV in the United States: Recommendations from the U.S. Department of Health and Human Services. MMWR Recomm. Rep. 2005, 54, 1-19. [PubMed]

8. Koblin, B.A.; Usher, D.; Nandi, V.; Tieu, H.V.; Bravo, E.; Lucy, D.; Miles, L.; Ortiz, G.; Kindlon, M.J.; Parisi, D.M.; et al. Postexposure Prophylaxis Awareness, Knowledge, Access and Use Among Three Populations in New York City, 2016-2017. AIDS Behav. 2018, 22, 2718-2732. [CrossRef]

9. Cardo, D.M.; Culver, D.H.; Ciesielski, C.A.; Srivastava, P.U.; Marcus, R.; Abiteboul, D.; Heptonstall, J.; Ippolito, G.; Lot, F.; McKibben, P.S.; et al. A case-control study of HIV seroconversion in health care workers after percutaneous exposure. Centers for Disease Control and Prevention Needlestick Surveillance Group. N. Engl. J. Med. 1997, 337, 1485-1490. [CrossRef] [PubMed]

10. Maxwell, S.; Shahmanesh, M.; Gafos, M. Chemsex behaviours among men who have sex with men: A systematic review of the literature. Int. J. Drug Policy 2019, 63, 74-89. [CrossRef] [PubMed]

11. Gonzales, R.; Mooney, L.; Rawson, R.A. The Methamphetamine Problem in the United States. Annu. Rev. Public Health 2010, 31, 385-398. [CrossRef]

12. Prakash, M.D.; Tangalakis, K.; Antonipillai, J.; Stojanovska, L.; Nurgali, K.; Apostolopoulos, V. Methamphetamine: Effects on the brain, gut and immune system. Pharmacol. Res. 2017, 120, 60-67. [CrossRef]

13. Halkitis, P.N.; Kapadia, F.; Siconolfi, D.E.; Moeller, R.W.; Figueroa, R.P.; Barton, S.C.; Blachman-Forshay, J. Individual, psychosocial, and social correlates of unprotected anal intercourse in a new generation of young men who have sex with men in New York City. Am. J. Public Health 2013, 103, 889-895. [CrossRef] [PubMed]

14. Substance Abuse and Mental Health Services Administration [SAMHSA]. National Survey on Drug Use and Health: Lesbian, Gay, $\mathcal{E}$ Bisexual (LGB) Adults; Center for Behavioral Health Statistics and Quality, Substance Abuse and Mental Health Services Administration: Rockville, MD, USA, 2019.

15. Braine, N.; Acker, C.J.; van Sluytman, L.; Friedman, S.; Des Jarlais, D.C. Drug use, community action, and public health: Gay men and crystal meth in NYC. Subst. Use Misuse 2011, 46, 368-380. [CrossRef] [PubMed] 
16. Rivera, A.V.; Harriman, G.; Carrillo, S.A.; Braunstein, S.L. Trends in Methamphetamine Use Among Men Who Have Sex with Men in New York City, 2004-2017. AIDS Behav. 2021, 25, 1210-1218. [CrossRef]

17. Garofalo, R.; Mustanski, B.S.; McKirnan, D.J.; Herrick, A.; Donenberg, G.R. Methamphetamine and young men who have sex with men: Understanding patterns and correlates of use and the association with HIV-related sexual risk. Arch. Pediatr. Adolesc. Med. 2007, 161, 591-596. [CrossRef] [PubMed]

18. Semple, S.J.; Zians, J.; Grant, I.; Patterson, T.L. Methamphetamine use, impulsivity, and sexual risk behavior among HIV-positive men who have sex with men. J. Addict. Dis. 2006, 25, 105-114. [CrossRef] [PubMed]

19. Halkitis, P.N.; Mukherjee, P.P.; Palamar, J.J. Longitudinal Modeling of Methamphetamine Use and Sexual Risk Behaviors in Gay and Bisexual Men. AIDS Behav. 2008, 13, 783. [CrossRef] [PubMed]

20. Cohen, S.E.; Vittinghoff, E.; Bacon, O.; Doblecki-Lewis, S.; Postle, B.S.; Feaster, D.J.; Matheson, T.; Trainor, N.; Blue, R.W.; Estrada, Y.; et al. High interest in preexposure prophylaxis among men who have sex with men at risk for HIV infection: Baseline data from the US PrEP demonstration project. J. Acquir. Immune. Defic. Syndr. 2015, 68, 439-448. [CrossRef] [PubMed]

21. McMahan, V.M.; Moreno, C.; Al-Tayyib, A.; Menza, T.W.; Orellana, E.R.; Bhattarai, A.; Stekler, J.D.; Glick, S.N. Pre-exposure Prophylaxis Awareness and Use Among Cisgender Men Who Have Sex With Men and Use Methamphetamine in 3 Western US Cities. Sex. Transm. Dis. 2020, 47, 217-223. [CrossRef]

22. Oldenburg, C.E.; Jain, S.; Mayer, K.H.; Mimiaga, M.J. Post-exposure prophylaxis use and recurrent exposure to HIV among men who have sex with men who use crystal methamphetamine. Drug Alcohol. Depend. 2015, 146, 75-80. [CrossRef]

23. Landovitz, R.J.; Fletcher, J.B.; Inzhakova, G.; Lake, J.E.; Shoptaw, S.; Reback, C.J. A novel combination HIV prevention strategy: Post-exposure prophylaxis with contingency management for substance abuse treatment among methamphetamine-using men who have sex with men. AIDS Patient Care STDs 2012, 26, 320-328. [CrossRef] [PubMed]

24. Reback, C.J.; Larkins, S.; Shoptaw, S. Methamphetamine abuse as a barrier to HIV medication adherence among gay and bisexual men. AIDS Care 2003, 15, 775-785. [CrossRef] [PubMed]

25. Freeman, P.; Walker, B.C.; Harris, D.R.; Garofalo, R.; Willard, N.; Ellen, J.M.; Adolescent Trials Network for HIV/AIDS Interventions 016b Team. Methamphetamine Use and Risk for HIV Among Young Men Who Have Sex With Men in 8 US Cities. Arch. Pediatr. Adolesc. Med. 2011, 165, 736-740. [CrossRef] [PubMed]

26. Halkitis, P.N.; Moeller, R.W.; Siconolfi, D.E.; Storholm, E.D.; Solomon, T.M.; Bub, K.L. Measurement model exploring a syndemic in emerging adult gay and bisexual men. AIDS Behav. 2013, 17, 662-673. [CrossRef] [PubMed]

27. Sobell, L.C.; Sobell, M.B. Timeline Follow-Back. In Measuring Alcohol Consumption: Psychosocial and Biochemical Methods; Litten, R.Z., Allen, J.P., Eds.; Humana Press: Totowa, NJ, USA, 1992; pp. 41-72.

28. Kinsey, A.C.; Pomeroy, W.R.; Martin, C.E. Sexual Behavior in the Human Male. Am. J. Public Health 2003, 93, 894-898. [CrossRef] [PubMed]

29. D'Avanzo, P.A.; Barton, S.C.; Kapadia, F.; Halkitis, P.N. Personality and its Relation to Mental and Psychosocial Health in Emerging Adult Sexual Minority Men: The P18 Cohort Study. Behav. Med. 2017, 43, 191-199. [CrossRef] [PubMed]

30. Jerome, R.C.; Halkitis, P.N.; Coley, M.A.; The Hope Team. Methamphetamine use patterns among urban Black men who have sex with men. Cult. Health Sex. 2009, 11, 399-413. [CrossRef] [PubMed]

31. Jerome, R.C.; Halkitis, P.N. Stigmatization, Stress, and the Search for Belonging in Black Men Who Have Sex With Men Who Use Methamphetamine. J. Black Psychol. 2009, 35, 343-365. [CrossRef]

32. Halkitis, P.N.; Levy, M.D.; Moreira, A.D.; Ferrusi, C.N. Crystal Methamphetamine Use and HIV Transmission Among Gay and Bisexual Men. Curr. Addict. Rep. 2014, 1, 206-213. [CrossRef] 